\title{
Editorial: Tribology in Snow and Ice Environments
}

\author{
Igor Velkavrh ${ }^{1 *}$, Werner Nachbauer ${ }^{2}$ and Thomas G. Mathia ${ }^{3}$ \\ ${ }^{1} \mathrm{~V}$-Research GmbH, Dornbirn, Austria, ${ }^{2}$ Department of Sport Science, University of Innsbruck, Innsbruck, Austria, ${ }^{3} \mathrm{UMR5513}$ \\ Laboratoire de Tribologie et Dynamique des Systèmes (LTDS), Écully, France
}

Keywords: friction mechanisms, cold environment, winter sports, ice, snow, tribology

\author{
Editorial on the Research Topic
}

\section{Tribology in Snow and Ice Environments}

Tribology in Snow and Ice Environments belongs to the most unknown parts of Tribology as the science of friction and wear. There are several reasons for it: the environment is hostile, the cost of on-site experimental investigations is very high and in a great number of cases results are confidential due to the competitive nature of the target applications in fields such as professional sports, automotive, aerospace, or naval industries as well as military. The dissemination of scientific findings is therefore limited. At the same time, snow and ice friction represent some of the most complex tribological phenomena with ambiguities in the understanding of the basic friction mechanisms. This is due to a myriad of influencing parameters, the unstable structure of snow and ice and the relatively vaguely understood properties of the interface that enables their low friction properties. Scientists are confronted with a multi-scale multi-disciplinary approach involving physics, mechanics, meteorology, rheology, kinesiology, geology, nivology, glaciology, and mathematics of transitional irreversible phenomena. In the last decade, new theoretical and experimental findings have challenged the existing theories and postulated a need for complete reformulation of the frameworks describing snow and ice friction. At present, novel hypotheses were put forward, which are supported by advanced analytical models and experimental procedures, but they are scattered throughout the literature.

This Research Topic on "Tribology in Snow and Ice Environments" collects the last developments in the field, written by researchers who have contributed significantly to the characterization of the properties of snow and ice concerning their friction mechanisms. The articles address various areas of the respective field but can roughly be ordered into three main groups: 1) topography and the calculation and measurement of the real contact area for ice and snow surfaces, 2) the use of specialized test-rigs for measuring of friction and tribological effects in snow and ice environments and 3) review of existing mechanisms.

Scherge et al. present the results of sliding tests with five typical grinding structures and compare them to calculations of the real area of contact. The measured ski base profiles and the measured grain size distribution of granular snow were employed within a bearing model for a rough surface in contact with loose and freely moving snow grains treated as ice spheres. For the analyzed structures, this model revealed a good correlation of the real area of contact between ski and snow with run times in lab-condition sliding tests. The results indicate that the snowcontaining volume of the grinding structure is pivotal for tailoring the sliding behavior. Lungevics et al. provide a review of the existing surface measurement methods related to ice tribology and present a holistic approach towards surface topography measurements for ice tribology applications. The entire sample surfaces are scanned, and the measured data is analyzed on different magnitude levels. This approach was applied to steel samples with different topographies which were tested on two different ice tribometers. A more 
straightforward correlation between the sample surface topography and its ice friction response was enabled. Brown provides explanations of how to apply recent advances in surface metrology science to understanding friction with snow and ice. Building on four decades of research using multiscale methods, in his article, two new surface metrology axioms and corollaries are proposed and potential applications of multiscale characterizations and analyses with good potential to provide new technological insights are provided.

Lemmettylä et al. present a custom-made ski tester, that was developed for measuring of friction of skis on real snow under laboratory conditions. Their ski tester is capable of glide testing cross-country and skate skis as well as kick simulation for the testing of grip waxes. In the presented study, glide testing precision was completed under three different conditions. The ski tester can distinguish the frictional differences between cross-country and skate skis under gliding and kick testing conditions. However, certain limitations due to the variations caused by the track preparation process need to be considered. Wolfsperger et al. present a novel approach towards the calculation of the speed of freestyle skiers or snowboarders during the in-run of a jump. Their work combines kinematic athlete data and comprehensive snow surface measurements to infer the coefficient of friction of freestyle skis and snowboards across a wide range of snow conditions. The air drag and lift of athletes were deployed from wind tunnel measurements. To solve the equation of motion for the coefficient of friction between ski/snowboard and snow, a mechanical model of the athlete was established. In their work, Hasler et al. aim to find indications or evidence for the presence of frictional meltwater in a ski/snow contact. The friction between the snow and a cross-country as well as a flat ski is measured on a largescale linear snow tribometer at realistic skiing speeds from 5 to $25 \mathrm{~m} /$ s. An infrared camera is used to analyze the snow temperature behind the skis. From the maximum snow surface temperature, the temperature at the spots where ski and snow are contacted are estimated. Heat flow considerations lead to the conclusion that more energy is dissipated into ski and snow than measured with heat flow. The most obvious mechanism for the additional energy dissipation is through snow melting. A second explanation may be provided by deformations of the snow bulk or ice grains; however, a detailed analysis of the energy consumption paths is currently not possible.

In their work, Lever et al. aim to assess our knowledge of the mechanics underlying ice and snow friction. In the article, a summary of the mechanical behavior of ice and snow substrates, behavior which perhaps has not received sufficient attention in friction studies is first presented. Afterwards, the strengths and weaknesses of five ice- and snow-friction hypotheses: pressuremelting, self-lubrication, quasi-liquid layers, abrasion, and icerich slurries are assessed. Their assumptions are discussed and evidence to determine whether they are consistent with the postulated mechanics reviewed. Lastly, the key issues that warrant additional research to resolve the specific mechanics and the transitions between them that control ice and snow friction across regimes of practical interest are identified.

We hope you will enjoy this Research Topic and we wish you plenty of reading pleasure on the latest findings in "Tribology in Snow and Ice Environments".

\section{AUTHOR CONTRIBUTIONS}

All authors contributed to the conception and design of the editorial.

Conflict of Interest: IV is employed by the company V-Research GmbH.

The remaining authors declare that the research was conducted in the absence of any commercial or financial relationships that could be construed as a potential conflict of interest.

Publisher's Note: All claims expressed in this article are solely those of the authors and do not necessarily represent those of their affiliated organizations, or those of the publisher, the editors and the reviewers. Any product that may be evaluated in this article, or claim that may be made by its manufacturer, is not guaranteed or endorsed by the publisher.

Copyright () 2022 Velkavrh, Nachbauer and Mathia. This is an open-access article distributed under the terms of the Creative Commons Attribution License (CC BY). The use, distribution or reproduction in other forums is permitted, provided the original author $(s)$ and the copyright owner(s) are credited and that the original publication in this journal is cited, in accordance with accepted academic practice. No use, distribution or reproduction is permitted which does not comply with these terms. 